Recepción: 22 / 02 / 2017

Aceptación: 14 / 04 / 2018

Ciencias económicas y empresariales

Publicación: 08 / 05 / 2018

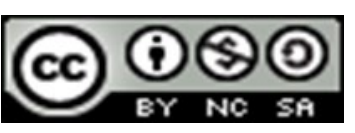

Artículo de Investigación

\title{
Calidad de vida laboral en los trabajadores del sector informal en Ecuador
}

\section{Quality of working life in informal sector workers in Ecuador}

\section{Qualidade de vida no trabalho dos trabalhadores do setor informal no Equador}

\author{
Monis R. Mendoza-Andrade ${ }^{\mathrm{I}}$ \\ monismendoza@hotmail.com \\ Arsenio H. Lucas-Muentes II \\ arse.lucas@hotmail.com \\ José V. Lucas-Muentes III \\ joselucas@hotmail.com
}

Correspondencia: arse.lucas@hotmail.com

\begin{abstract}
${ }^{\text {I }}$ Magister en Desarrollo Humano y Comunitario, Licenciado en Trabajo Social, Docente de la Universidad Técnica Luis Vargas Torres de Esmeraldas, Esmeraldas, Ecuador.

II Master en Ciencias Especialidad Docencia Universitaria, Licenciado en Ciencias de la Educación; Profesor de Enseñanza Secundaria en la Especialización de Físico Matemáticos, Doctor en Ciencias De La Educación Mención Enseñanza de la Matemática, Docente de la Universidad Técnica Luis Vargas Torres de Esmeraldas, Esmeraldas, Ecuador.

${ }^{\text {III }}$ Master en Ciencias Especialidad Docencia Universitaria, Doctor en Ciencias de la Educación Mención Enseñanza de la Matemática, Licenciado en Ciencias de la Educación Profesor de Enseñanza Secundaria en la Especialización de Físico Matemáticas, Docente de la Universidad Técnica Luis Vargas Torres de Esmeraldas, Esmeraldas, Ecuador.
\end{abstract}




\section{Resumen}

La calidad de vida es un tema complejo al tratarse aspectos diversos del ser humano, su familia y contexto, si además le agregamos la categoría trabajo desarrollada desde el sector laboral informal, las dimensiones se magnifican, por ser el trabajo un concepto múltiple con gran variedad de indicadores involucrados, entre los cuales se encuentran los niveles de ingreso, salud ocupacional, satisfacción en el desempeño de la labor, calidad del ambiente laboral, motivación, identificación organizacional, bienestar de los trabajadores, seguridad social para el trabajador y sus familiares, jubilación, beneficios de ley, concepción positiva del trabajo, entre otros. Es por eso que se realiza esta investigación bajo el enfoque cualitativo, siguiendo el método Investigación Acción, aplicando técnicas de recolección de información como la entrevista, el cuestionario, los grupos de discusión y diarios de campo. Los informantes claves están representados por tres (03) mujeres trabajadoras informales, de la ciudad de Quito. Entre las técnicas para el análisis de la información se emplearon la categorización, la estructuración, la triangulación y contrastación. Los resultados dan cuenta de la necesidad de un cambio sustancial en los mecanismos, para optar y mantenerse en un empleo formal, vistos desde un enfoque holístico que respete la idiosincrasia de los pueblos.

Palabras clave: Calidad de vida laboral; trabajo informal; salud ocupacional.

\section{Abstract}

The quality of life is a complex issue to deal with different aspects of the human being, his family and context, if we also add the category work developed from the informal labor sector, the dimensions are magnified, since the work is a multiple concept with great variety of involved indicators, among which are the levels of income, occupational health, satisfaction in the performance of the work, quality of the work environment, motivation, organizational identification, welfare of workers, social security for the worker and their families, retirement, benefits of law, positive conception of work, among others. That is why this research is carried out under the qualitative approach, following the Action Research method, applying information gathering techniques such as interview, questionnaire, discussion groups and field journals. The key informants are represented by three (03) informal women workers from the city of Quito. Among the techniques for the analysis of information, categorization, structuring, triangulation and contrast were used. The results show the need for a substantial change in the mechanisms, to 
opt for and maintain a formal employment, seen from a holistic approach that respects the idiosyncrasies of the peoples.

Keywords: Quality of working life; informal work; occupational health.

\section{Resumo}

A qualidade de vida é uma questão complexa para lidar com diferentes aspectos do ser humano, sua família e contexto, se somarmos a categoria trabalho desenvolvido a partir do trabalho informal, as dimensões são ampliadas, pois o trabalho é um conceito múltiplo com grande variedade dos indicadores envolvidos, entre os quais estão os níveis de renda, saúde ocupacional, satisfação no desempenho do trabalho, qualidade do ambiente de trabalho, motivação, identificação organizacional, bem-estar dos trabalhadores, segurança social para o trabalhador e suas famílias, aposentadoria, benefícios da lei, concepção positiva do trabalho, entre outros. É por isso que esta pesquisa é realizada sob a abordagem qualitativa, seguindo o método de Pesquisa-Ação, aplicando técnicas de coleta de informações, como entrevista, questionário, grupos de discussão e periódicos de campo. Os informantes-chave são representados por três (03) trabalhadoras informais da cidade de Quito. Entre as técnicas para análise de informação, categorização, estruturação, triangulação e contraste foram utilizadas. Os resultados mostram a necessidade de uma mudança substancial nos mecanismos, de optar e manter um emprego formal, visto a partir de uma abordagem holística que respeite as idiossincrasias dos povos.

Palavras chave: Qualidade de vida no trabalho; trabalho informal; saúde ocupacional.

\section{Introducción}

Nuestro Banco Central, publicó las cuentas nacionales sobre el crecimiento del país, cuyas cifras dan cuenta que la economía ecuatoriana (PIB) en el año 2017 registró un crecimiento de 3.0\%. Lo que se traduce en un comportamiento que revela un dinamismo en la economía y que tiene su explicación en la evidencia del aumento del Gasto del Consumo Final de los Hogares, el Gasto de Consumo Final del Gobierno General y las Exportaciones. De esta manera, el PIB alcanzó \$103.057 millones. Teniendo el Gasto de Consumo Final de los Hogares una tasa de variación positiva de 4.9\%, con una contribución al crecimiento del PIB de 2.96 puntos porcentuales a la variación anual del PIB. Mostrando que el Gasto de Consumo Final del Gobierno General, en el 
año 2017, registró un crecimiento de 3.8\% con relación al año anterior, contribuyendo positivamente a la variación del PIB en 0.56 puntos porcentuales, dijo la entidad (INEC, 2017).

Incluso el propio Fondo Monetario Internacional (FMI) dijo estar sorprendido por el desempeño económico de Ecuador, del cual esperaba, según un estudio de octubre pasado, un crecimiento de apenas el 0,2 por ciento. Se trata de una economía que "nos ha sorprendido en el año 2017 de manera favorable, con un crecimiento mayor al que habíamos anticipado", admitió a finales de noviembre el director del Departamento Occidental del FMI, Alejandro Werner, tras un encuentro en Quito con altas autoridades del Gobierno.

Sin embargo, el tema que incentivo esta investigación es la calidad de vida laboral de los trabajadores del sector informal, cuya existencia es innegable y que se evidencia en nuestros informantes claves constituidos por tres emprendedoras mujeres que asisten diariamente a ganarse el sustento para ellas y sus familias, de manera informal en la ciudad de Quito y que a través de sus vivencias experiencias y saberes muestran como es la vida del trabajador, que no goza de los beneficios de Ley y que sabe que con el paso del tiempo será más difícil integrarse a la formalidad, por la edad y la escasa o nula formación profesional que poseen, aunado a la preocupación en unos casos y en otros a la tranquilidad que quizás sus hijas e hijos tengan el mismo futuro con pocas posibilidades de trabajo formal. Reconocen que son las mujeres quienes integran mayormente este grupo de informales, aunque por disposición del artículo 331 de la Constitución de la República, es deber del Estado garantizar a las mujeres igualdad en el acceso al empleo, a la formación y promoción laboral, profesional y a la remuneración equitativa y que dicho articulado promueve la adopción de todas las medidas necesarias para eliminar las desigualdades, además que se prohíbe toda forma de discriminación, acoso o acto de violencia de cualquier índole, sea directa o indirecta, que afecte a las mujeres en el trabajo (Asamblea Constituyente de Ecuador, 2008)

Es importante revisar que la informalidad laboral en Latinoamérica no se distribuye de forma homogénea. En países con una alta renta per cápita como Argentina, Uruguay, Brasil, Panamá o Chile es sustancialmente menor que en Centroamérica, donde puede llegar al 70.7\% de los trabajadores, como en el caso de Honduras, (OIT, 2012). En cuanto a la composición de género, el $45 \%$ de los hombres y el $55 \%$ de las mujeres trabajan en condición informal. Paralelamente, 
afecta al 56\% de los jóvenes de 15 a 24 años. Pero esta condición está arraigada en el Ecuador y la región por varios factores. Aunque la afiliación de las empleadas de servicio doméstico a la seguridad social constituyó un paso importante en la formalización laboral en el Ecuador, en sectores como el comercio y la construcción, hay pendientes. Las ramas de actividad con mayores tasas de empleo informal en la región son la construcción y el comercio. González sostiene que la falta de oportunidades laborales obedece al entorno económico en las grandes ciudades que son las más pobladas y con mayor migración rural, pero también inciden los niveles de educación. A menor educación peores condiciones laborales (González, 2017).

En esta investigación se considera a la informalidad como una manifestación de las relaciones entre los agentes económicos y el Estado que, según la literatura económica, desempeña una función importante en cuanto a mitigar las fallas del mercado, asegurar el suministro de bienes públicos, y mantener condiciones para la igualdad de oportunidades (Perry, 2007). Sin embargo, también se destaca otra mirada ubicada en el concepto de escape (“exit") de (Hirschman, 1970), en el cual se plantea que muchos trabajadores, empresas y familias escogen su nivel óptimo de adherencia con los mandatos y las instituciones del Estado, en concordancia o no con el valor que asignen a los beneficios netos relacionados con la formalidad y al esfuerzo y la capacidad de fiscalización del Estado. Es decir, analizan los costos-beneficios de insertarse en la formalidad, y generalmente prefieren no hacerlo. Por lo tanto, en esta mirada, los altos niveles de informalidad son una consecuencia de que un gran número de empresas y personas optan por no pertenecer a las instituciones formales. (Hirschman, 1970)

Es importante acotar, que en nuestro país la Ley y especialmente la Constitución de la República en su artículo 33, establece que el trabajo es un derecho y un deber social, y un derecho económico, fuente de realización personal y base de la economía. El Estado garantizará a las personas trabajadoras el pleno respeto a su dignidad, una vida decorosa, remuneraciones y retribuciones justas y el desempeño de un trabajo saludable y libremente escogido o aceptado, igualmente el artículo 284 del mismo cuerpo constitucional, señala que la política económica tendrá, entre otros, el objetivo de impulsar el pleno empleo y valorar todas las formas de trabajo, con respeto a los derechos laborales, así como mantener la estabilidad económica, entendida como el máximo nivel de producción y empleo sostenibles en el tiempo. 
De acuerdo a la referida norma constitucional, toda persona tendrá derecho a desarrollar sus labores en un ambiente adecuado y propicio, que garantice su salud, integridad, seguridad, higiene y bienestar. Se evidencia lo tajante en cuanto al papel preponderante que tiene el trabajo para los Hombres y Mujeres en el país, haciendo énfasis en los beneficios sociales correspondientes a los derechos reconocidos de los trabajadores, de carácter obligatorio y que van más allá de la remuneración normal y periódica que deben recibir por su trabajo, entre ellas están la afiliación a la seguridad social, pago por horas extras y suplementarias, pago de la décimotercera (bono navideño) y decimocuarta remuneración (bono escolar), pago del fondo de reserva, vacaciones anuales, jubilación, licencia por maternidad/paternidad y pago de utilidades, la gran interrogante es ¿dónde quedan todos estos beneficios cuándo el trabajador o trabajadora está bajo el status de informal y cómo esto afecta su calidad de vida?.

El trabajador y trabajadora tienen, además de las necesidades básicas que todo ser humano manifiesta, una necesidad económica sustentada en la responsabilidad familiar, ya que son los proveedores del hogar donde generalmente existen varias generaciones cohabitando (hijos, padres, abuelos), en el caso de los trabajadores informales, en su mayoría vienen de una tradición familiar de no dependencia, con poca o escasa formación profesional, procedente de zonas rurales donde la idiosincrasia juega un papel preponderante y marca a estas persona en su cosmovisión de vida. Tenemos también en las zonas urbanas trabajadores que no se preparan formalmente para el desempeño de una profesión y otros que desarrollan un arte u oficio y que no sienten la necesidad de estar institucionalizados o que no llenan los requisitos para optar a empleos formales.

Recordemos que, el sector informal es considerado como un grupo de unidades de producción que, según las definiciones y clasificaciones del Sistema de Cuentas Nacionales de las Naciones Unidas, forman parte del sector de los hogares. Es decir, son empresas (unidades económicas) que pertenecen a los hogares y que no están constituidas en sociedad. En concordancia con esta definición, se tiene el empleo en el sector informal como el conjunto de personas ocupadas que trabajan en establecimientos que no se encuentran registrados ante la autoridad tributaria, es decir, en las empresas que no tienen Registro Único de Contribuyente (RUC) o que no tributa bajo el Régimen Impositivo Simplificado Ecuatoriano (Rise). Por su parte, el empleo en el sector formal lo componen las personas que trabajan en establecimientos que tienen RUC. Los 
informales, según la entidad, son quienes trabajan para o constituyen una empresa que no tiene Registro Único de Contribuyentes (RUC).

El Instituto Nacional de Estadística y Censo (INEC), atribuye la informalidad a quienes laboran en negocios que no tienen Registro Único de Contribuyentes. Si esta cifra se ha elevado es porque ha habido un traslado de las personas en desempleo a la informalidad, según la reflexión de López. "El que estaba desocupado ha puesto un fogón, está vendiendo en la calle... En definitiva, está tratando de sobrevivir, porque tampoco se puede morir de hambre", comenta el exgerente del Banco Central Ecuatoriano (López, 2017). Las personas que se encuentran en la informalidad están expuestas a condiciones de trabajo inseguras, tienen pocas oportunidades de formación, ingresos irregulares, jornadas de trabajo más extensas, no obtienen los beneficios laborales y no todos acceden a la seguridad social, "Se trata de una situación altamente vulnerable, porque el trabajo en la economía informal está efectivamente fuera del alcance de la legislación".

Esto trae consecuencias en varios ámbitos de la sociedad, en cuanto a la recaudación los negocios informales, que no declaran ingresos, merman la capacidad recaudatoria del Estado. Los negocios formales compiten en desigualdad con los informales que asumen menos costos. Las trabajadoras y trabajadores informales no son favorecidos con la cesantía, que es otra de las prestaciones a las que no pueden acceder los empleados informales al salir del trabajo, al igual que la Jubilación porque al no cotizar a la Seguridad Social, los trabajadores informales no tienen la prestación pública de este beneficio, no poseen seguro de salud, ni afiliación al Instituto Ecuatoriano de Seguridad Social (IESS) ni seguro médico privado corporativo. Deben contratar uno privado para la cobertura de salud, además, sin rol que respalde ingresos regulares, obstaculiza el acceso a crédito o hipoteca y entorpece el desarrollo de la persona, su familia y la nación, por supuesto que todo esto mina la calidad de vida del trabajador informal.

\section{Metodología}

Es importante mencionar que esta investigación surge para dar respuesta al planteamiento, de cómo es la calidad de vida de los trabajadores informales en la República de Ecuador, para eso se decidió hacer una investigación planteada desde sus actores, desde aquellos que viven su realidad y pueden dar testimonio de ella, constituyéndose en informantes claves que en esta oportunidad 
son tres mujeres trabajadoras informales. Se escogió el enfoque cualitativo porque nos permite entrar en el mundo del otro y le permite a él y ella empoderarse de su realidad para transformarla (Castillo, 2003), lo cual se logra con la corriente crítica que es, en palabras de Park en (Salazar, 2012) reflexionar sobre su realidad para que comprendan la necesidad de comprometerse en acciones que lo lleven a transformar su situación "Sólo cuando el hombre toma conciencia de su realidad puede convertirse en el agente y sujeto de los cambios que en ella se dan", de esta manera, dicha corriente tiene como finalidad la transformación de la estructura de las relaciones sociales y dar respuesta a determinados problemas generados por éstas.

Es por eso que la investigación se coloca dentro de un estudio de tipo acción participación, definido por (Bartolomé, 1986) como "un proceso reflexivo que vincula dinámicamente la investigación, la acción y la formación, realizada por profesionales de las ciencias sociales, acerca de su propia práctica. Se lleva a cabo en equipo, con o sin ayuda de un facilitador externo al grupo", así, en la investigación acción, el investigador se ocupa de interactuar con la gente que vive, siente y padece el fenómeno en estudio con el propósito de analizar sus causas, consecuencia y posibles soluciones.

En la investigación cualitativa, es fundamental la interacción con las personas que viven la problemática y una de las técnicas más empleadas es la entrevista, porque nos permite acercarnos y escuchar a profundidad a las personas involucradas en la situación objeto de investigación, siendo ésta definida por (Murillo, 2015), como "la técnica con la cual el investigador pretende obtener información de una forma oral y personalizada”. En este sentido, la información versará en torno a acontecimientos vividos y aspectos subjetivos de la persona tales como creencias, actitudes, opiniones o valores en relación con la situación que se está estudiando.

La aplicación de un método es el cumplimiento de pasos, que en este caso se rige por las distintas fases que proponen (Colas \& Buendia, 2012) para su desarrollo, siendo el diagnóstico y reconocimiento de la situación, el inicio, lo que nos permite reconocer la realidad en el lugar de los acontecimientos y en convivencia con los informantes claves, para luego diseñar el proyecto que marcara la pauta de la investigación; posteriormente avanzamos con el desarrollo de un Plan de Acción en sinergia a la propuesta de gestionar agrupaciones de mujeres trabajadoras informales, en torno a sus capacidades y campo de desempeño (alimentos, servicios, textiles, 
otros) buscando la capacitación y formación de estas mujeres para dar respuesta a las diferentes debilidades diagnosticadas el mundo laboral o del emprendimiento, así como incentivarlas a continuar con sus estudios formales.

Seguidamente, mediante un cuestionario, se abordaron trabajadores del municipio para conocer su postura y comportamiento frente a los trabajadores y trabajadoras informales y se les pidió su colaboración en propuestas para mejorar la comunicación entre ambos.

Llegamos a la evaluación, donde se aplica el análisis crítico y reflexivo de cada acción, mediante la constante evaluación, y autoevaluación de las estrategias utilizadas por parte de todos los participantes en el proceso de investigación-acción, además de la revisión exhaustiva de los diarios de campo aplicados a los grupos de discusión, para conocer los alcances de la experiencia y el grado de cumplimiento de los objetivos.

Finalizamos con la aplicación del análisis, la reflexión, la crítica y auto critica que siempre estuvieron presentes en el proceso, pero que en esta etapa se hacen más evidentes y necesarias, para pasar a la elaboración de las conclusiones que darán pie al reinicio del ciclo espiral, de donde surgen nuevas propuestas, los inconvenientes y los aportes de todos los involucrados.

Para procesar la información, se aplicaron las técnicas de categorización, codificación, triangulación, y contrastación.

\section{Resultados}

Las mujeres y los jóvenes son los que se insertan en mayor proporción al trabajo informal, seguidos de los mayores de 50 años que ya perdieron un empleo formal.

La mayoría de trabajadores informales están casados o en unión libre, y son jefes de hogar.

Las empresas vinculadas con el sector informal, se dedican en su mayoría al negocio de licores, confecciones y textiles, agricultura, productos artesanales o tecnológicos y al traslado de mercancías a través de las fronteras.

El trabajo informal es una amenaza para las empresas que cumplen la Ley, porque no pagan impuestos, pero más para las mujeres y hombres que laboran en ellas, porque no los capacitan, se desconoce si emplean menores de edad, se duda de que gocen de mano de obra calificada porque 
demandaría un mayor sueldo. Ni siquiera se conoce si pagan, por lo menos, el sueldo básico o cumplen las cuarenta (40) horas de trabajo, que están establecidas en la normativa del Ecuador.

Los ingresos son menores, variables e irregulares, haciendo que las condiciones de los trabajadores del sector informal sean más difíciles, que el resto de los empleados.

Los trabajadores por cuenta propia son los que desarrollan su actividad utilizando su trabajo personal, aunque pueden estar ayudados por familiares no remunerados, lo que incrementa las dificultades económicas del grupo familiar.

Es prácticamente imposible para este tipo de trabajador acogerse a las regulaciones sobre remuneraciones mínimas ni seguridad social, porque su cotidianidad lo obliga a vivir día a día, ingresando lo mínimo para vivir, por lo tanto, su calidad de vida es escueta, ya que no goza de estabilidad, ni vacaciones, ni otro tipo de beneficios, en incumplimiento total de sus derechos.

\section{Recomendaciones}

Es indispensable que se sigan creando, ejecutando y haciendo seguimiento y control de las políticas públicas para mejorar las condiciones laborales, que enfrentan las personas inmersas en el trabajo informal, así como incentivar y apoyar a los protagonistas de la economía formal (industrias, comercios, servicios) para la creación en condiciones de equidad, respeto y humanismo de puestos de trabajo formales.

Se deben hacer campañas de capacitación y formación que les permitan a los trabajadores informales, mejorar sus competencias, para que puedan dominar aspectos como la tributación, ventas, contabilidad básica, manipulación y presentación de los alimentos con base en condiciones de salubridad, legislación laboral, para que conozcan sus derechos y deberes formales, así como asesoría legal para instruirse en las exigencias establecidas por el municipio para los trabajadores informales y así puedan cumplirlas a cabalidad.

No se puede ver la formalidad solo desde el ámbito del pago de tributos, se debe garantizar la calidad de vida de los trabajadores, sean formales o informales, porque en resumen son seres humanos buscando medios de subsistencia. 


\section{Referencias Bibliográficas}

Asamblea Constituyente de Ecuador. (28 de Septiembre de 2008). Constitución de la República

del Ecuador. Quito, Ecuador: Asamblea Constituyente de Ecuador.

Bartolomé, M. (1986). La investigación cooperativa. Educar (10), 51-79.

Castillo, E. (2003). Lo científico del a investigación cualitativa: Viejos dilemas, nuevas posturas.

Nómadas, 40.

Colas, M., \& Buendia, L. (2012). Investigación educativa. Profesorado, 83.

González, M. (16 de Julio de 2017). La informalidad laboral, una condición arraigada en Ecuador. Líderes, pág. 43.

Hirschman, A. (1970). Exit Voice and Loyalty: Reponses to Decline in Firms, Organizations and States. Cambridge: Harvard University Press.

INEC. (2017). Reporte de economia laboral. Quito: Dirección de Estudios y Análisis de la Información.

López, M. (2017). Entrevista. Ecuador: Expreso. ec.

Murillo, J. (2015). La entrevista. Slideshare, 21.

OIT. (2012). Panorama Laboral 2012, América Latina y el Caribe. Lima: Organización Internacional del Trabajo.

Perry, G. E. (2007). Informalidad. Washington, DC 20433: The International Bank for Reconstruction and Development / The World Bank.

Salazar, M. (2012). La Investigación-acción-participativa. Inicios y desarrollos. EDUCERE, 394. 